\title{
Mesenteric Lymph Duct Ligation Alleviates Acute Lung Injury Caused by Severe Acute Pancreatitis Through Inhibition of High Mobility Group Box 1-Induced Inflammation in Rats
}

\author{
Yishuang Tang ${ }^{1} \cdot$ Jing Kong $^{1} \cdot$ Bingduo Zhou ${ }^{1}$ (D $\cdot$ Xiaosu Wang $^{1} \cdot$ Xiaowen Liu $^{1} \cdot$ Yi Wang $^{1} \cdot$ Shengliang Zhu ${ }^{1}$
}

Received: 12 August 2020 / Accepted: 16 December 2020 / Published online: 12 January 2021

(C) The Author(s) 2021

\begin{abstract}
Background Acute lung injury (ALI) is the most common complication and one of the leading causes of mortality of severe acute pancreatitis (SAP). Nevertheless, no effective therapeutic schemes are presently available.

Aims To investigate the effect and potential mechanism of mesenteric lymph duct ligation (MLDL) on experimental SAPinduced ALI.

Methods Immediately following MLDL, rats were subjected to SAP by retrograde injection of $5 \%$ sodium taurocholate into the biliopancreatic duct. At $24 \mathrm{~h}$ after modeling, tissues were collected for morphological examination. The levels of TNF$\alpha$, IL-6, intercellular adhesion molecule-1 (ICAM1), diamine oxidase (DAO), and D-lactic acid (D-LA) in serum, and the myeloperoxidase (MPO) activity in lung tissues were determined. Moreover, the expressions of high mobility group box 1 (HMGB1), receptor of advanced glycation endproducts (RAGE), and NF- $\mathrm{kB}$ p65 at the mRNA and protein levels in lung tissues, and the expressions of HMGB1, RAGE, and TNF- $\alpha$ at the mRNA level in intestinal lymphoid tissues were evaluated. Results MLDL significantly attenuated the histological injury of the pancreas and lung and reduced the production of TNF- $\alpha$, IL-6, and ICAM1. Besides, MLDL repressed the activity of MPO in the lung. However, the levels of serum DAO and DLA were decreased without obvious morphological improvement in intestinal injury. Moreover, MLDL apparently reduced the up-regulation of HMGB1, RAGE, and NF- $\mathrm{BB}$ p65 in lung tissues, as well as the expressions of HMGB1, RAGE, and TNF- $\alpha$ in intestinal lymphoid tissues.

Conclusions Mesenteric lymph was a source of harmful factors leading to SAP-ALI. MLDL could alleviate SAP-ALI probably by inhibiting HMGB1-induced production of inflammation factors.
\end{abstract}

Keywords Mesenteric lymph $\cdot$ Acute lung injury $\cdot$ Severe acute pancreatitis $\cdot$ HMGB1

\section{Introduction}

Acute pancreatitis (AP) is a common inflammatory disease caused by multiple factors, which can result in an approximately $35 \%$ mortality when progressing into severe acute

Supplementary Information The online version contains supplementary material available at https://doi.org/10.1007/s1062 0-020-06801-6.

Bingduo Zhou

bingduozhou@163.com

1 Department of Gastroenterology, Yueyang Hospital of Integrated Traditional Chinese and Western Medicine, Shanghai University of Traditional Chinese Medicine, Shanghai, China pancreatitis (SAP) [1, 2]. SAP is characterized by systemic inflammatory responses (SIRS) and multiple organ dysfunction syndromes (MODS). The lung is the most susceptible organ to inflammation, and acute lung injury (ALI) is the most common remote organ damage occurring in at least 10-25\% SAP cases [3, 4], which may develop into MODS and is responsible for $30-40 \%$ SAP-associated deaths [5-7]. Despite the prevalence of ALI and its key contribution to SAP, no effective therapeutic schemes are presently available.

It is now well established that the intestinal barrier of SAP patients is often damaged, allowing intestinal bacteria and endotoxins to invade the blood circulation, which subsequently leads to clinical worsening, including MODS or even death [8]. It is also worth noting that despite the sequence of organ failure in MODS may vary, the lung is 
the commonest organ in organ failure and generally the first one to fail clinically in SAP $[9,10]$. However, mechanisms underlying the injured intestine and lung remain not fully understood.

In recent years, researchers have pay attention to mesenteric lymph, which follows a direct anatomical path between the gut and bloodstream. Mesenteric lymph continuously drains from the mesenteric lymphatics to the thoracic duct, subsequently merging with the blood circulation in the left subclavian vein. Thence, the lung is the first capillary bed encountering mesenteric lymph and the first organ in contact with gut-derived products drained by mesenteric lymph [11]. The anatomic basis partly explains the clinical observation that the lung is generally the first organ to fail in some critical illnesses. In experimental models of hemorrhagic shock and trauma, interrupting the flow of mesenteric lymph into systemic circulation by selective mesenteric lymph duct ligation (MLDL) or mesenteric lymph drainage offers some protective effects against organ failure [12-14], especially respiratory dysfunction $[15,16]$, suggesting that factors released from the injured intestine and carried in the mesenteric lymph significantly contribute to the development of organ dysfunction. Therefore, identifying and blocking the translocation of gut-derived toxic mediators through mesenteric lymph seems to be a tempting strategy for future therapeutics of ALI.

The release of inflammatory mediators plays a key role in the pathogenesis of ALI. Though several mediators, such as TNF- $\alpha$, IL- $1 \beta$, and IL- 6 , have been frequently investigated, the other mediators that also play a prominent role in inflammation, such as high mobility group box 1 (HMGB1), have not been fully elucidated. HMGB 1 is a highly conserved, nuclear protein present in all cell types that can be actively secreted into extracellular spaces in response to external stimuli, or passively released from necrotic cells [17]. Advanced glycation endproducts (RAGE) have been fully confirmed to act as one of the main HMGB1 receptors [17]. Once HMGB 1 binds to RAGE, the intracellular signal transduction, such as NF-kB pathways, will be promoted, which will trigger the release of various cytokines, such as TNF- $\alpha$, IL- $1 \beta$, and IL- 6 , leading to increased tissue injury and organ dysfunction $[18,19]$. As an important pro-inflammatory mediator, HMGB 1 has been associated with the onset and progression of a wide range of disorders, including ALI [20]. It also has been previously reported that the circulating HMGB1 level is significantly increased in SAP patients within $72 \mathrm{~h}$ after the disease onset, and it is correlated with the disease severity [21, 22]. In experimental SAP animals, serum, and pancreatic tissues, the HMGB1 level is increased at 6-12 h after model establishment [23, 24], indicating the proinflammatory role of HMGB1 in the progression of SAP.
In the present study, we explored whether blocking the flow of mesenteric lymph into systemic circulation by MLDL could protect lung tissue against HMGB1-mediated inflammatory injury. Collectively, our findings provided valuable insights into new treatment options of SAP-ALI.

\section{Materials and Methods}

All the experimental protocols were approved by the Committee on Ethics of Animal Experiments of Shanghai University of Traditional Chinese Medicine, and animal-related experiments were carried out following the principles outlined in the National Institutes of Health Guide for the Care and Use of Laboratory Animals.

\section{Animals}

A total of 45 healthy Sprague-Dawley rats (male, 8 weeks old, $200 \pm 20 \mathrm{~g}$ ) were purchased from Shanghai SLAC Laboratory Animal Co., Ltd. (China) and housed in an environmentally controlled room at $20 \pm 2{ }^{\circ} \mathrm{C}$ with a relative humidity of $45-65 \%$ under a 12:12-h light/dark cycle. All animals were given free access to standard laboratory food and water for 1 week.

\section{Modeling and Grouping}

The rats were randomly allocated into three groups $(n=15$ per group) as follows: the sham operation group (SO), the SAP group (SAP), and the MLDL group (MLDL). Rats were anesthetized with $2.0 \%$ pentobarbital sodium $(2 \mathrm{~mL} / \mathrm{kg}$, Sinopharm Chemical Reagent Co., Ltd., China, WS20171129) in an induction chamber following 12-h fasting. Subsequently, the pancreas was exposed along a midline incision, and the biliopancreatic duct was cannulated through the duodenum. The hepatic duct was temporarily closed by a microvascular clamp. The SAP model was induced by a standard retrograde infusion of $5.0 \%$ sodium taurocholate (STC, Sigma, St. Louis, MO, USA, CAS145-42-6) solution $(1 \mathrm{mg} / \mathrm{kg})$ at a constant rate of $12 \mathrm{~mL} / \mathrm{h}$ using an infusion pump into the bile-pancreatic duct. After this procedure, the pancreas was carefully put back in place, and the abdomen was closed. In the SO group, rats received sham operation, meaning that the pancreas was only flipped instead of STC infusion. In the MLDL group, the mesenteric lymph ducts were ligated before modeling. For MLDL, the rats were anesthetized, and then a long midline incision was made. The efferent mesenteric lymphatic vessel, which lies adjacent to the superior mesenteric artery (SMA), was identified by reflecting the loops of the intestine to the left of the animal with moist gauze swabs. Next, the mesenteric 
lymphatic duct was isolated and double ligated with a 5-0 silk tie to ensure that the mesenteric lymph was completely blocked. The rats were then subjected to SAP modeling as described above.

\section{Sample Collection}

At $24 \mathrm{~h}$ after SAP modeling, the rats were anesthetized by intraperitoneal injection of $2.0 \%$ pentobarbital sodium, and the chests were opened quickly. Blood samples were obtained from the abdominal aorta, followed by centrifugation at $1200 \mathrm{~g}$ for $15 \mathrm{~min}$ at $4{ }^{\circ} \mathrm{C}$. The serum was cryopreserved at $-80^{\circ} \mathrm{C}$. Next, the rats were euthanized under anesthesia. Pancreatic, pulmonary, and terminal ileum tissues, as well as mesenteric lymph tissues, were harvested and preprocessed for the following experiments.

\section{Histopathology Analysis}

Pancreatic heads, the right upper lung, and terminal ileum tissues were harvested, trimmed, and fixed in $4 \%$ neutral buffered paraformaldehyde overnight. The fixed tissue specimens were embedded in paraffin, sequentially sliced into 4- $\mu \mathrm{m}$ sections, and then stained with hematoxylin and eosin (H\&E) solution. For all groups, histological sections were graded by two experienced pathologists who were blind to the experimental protocol under a light microscope at $200 \times$ magnification. The severity of the pancreatic, lung and intestinal injury was then evaluated as previously described by Kubisch [25], Imanaka [26], and Crissinger [27]. Finally, the total histopathology scores were calculated and compared.

\section{Analysis of Myeloperoxidase (MPO) in Lung Samples}

Lung tissue samples were thawed, homogenized in $20 \mathrm{mM}$ phosphate-buffered saline (PBS) $(\mathrm{pH}=7.4)$, and centrifuged at $1200 \mathrm{~g}$ for $20 \mathrm{~min}$ at $4{ }^{\circ} \mathrm{C}$. The supernatant was assayed for MPO activity using enzyme-linked immunosorbent assay (ELISA) kits (Nanjing Jiancheng, China). All procedures were conducted according to the manufacturer's instructions.

\section{Measurement of Biochemical Indexes in Serum}

The concentrations of serum TNF- $\alpha$, IL-6, ICAM1, DAO, and D-LA were determined using commercially available ELISA kits (Nanjing Jiancheng, China) according to the manufacturer's instructions. Plates were read using a microplate reader (Multiskan MK3, Finland) at a wavelength of $450 \mathrm{~nm}$.

\section{Real-Time PCR (RT-PCR)}

Total RNA was extracted with TRIZOL reagent (Invitrogen, Carlsbad, CA, USA) and reversely transcribed into cDNA. RT-PCR was performed using an ABI7500 Fast Real-time PCR machine. Briefly, the amplifications were carried out with 40 cycles at a melting temperature of $95^{\circ} \mathrm{C}$ for $3 \mathrm{~s}$ and an annealing temperature of $60^{\circ} \mathrm{C}$ for $30 \mathrm{~s}$. The specific primers were designed and synthesized by Sangon Biotech (Shanghai, China), and the primer sequences were as follows: HMGB1, forward 5'- CGC GGAGGAAAATCAACTAA-3' and reverse 5'-GGGTGC TTCTTCTTGTGCTC-3'; RAGE forward: 5'-AGGAAC GTGCAGAGCTGAAT-3', reverse 5'-AGAAAGTGGCTC GAGGTTGA-3'; NF- $\mathrm{kB}$ p65 forward: 5'-GCGTTTCCG TTACAAGTGCG-3', reverse 5'- GTGCGTCTTAGTGGT ATCTGTGC-3'; TNF- $\alpha$ forward: 5'-TGCCTCAGCCTC TTCTCATT- $3^{\prime}$, reverse $5^{\prime}$-CCCATTTGGGAACTTCTC CT-3'; $\beta$-actin forward: 5'-AGCCATGTACGTAGCCAT CC-3', reverse: 5'-ACCCTCATAGATGGGCACAG-3'. The relative expressions of target genes were calculated by the $2^{-\Delta \Delta \mathrm{Ct}}$ method.

\section{Western Blotting Analysis}

Total proteins were extracted from the lung tissues using a whole protein extraction kit (KeyGen Biotech Co., Ltd., Nanjing, China), and a BCA protein assay kit (Thermo, USA, PICPI23223) was adopted to determine the protein concentration. Briefly, equal amounts of proteins $(50 \mu \mathrm{g})$ were subjected to SDS-PAGE on $10 \%$ gels and then transferred onto a polyvinylidene fluoride membrane. The membranes were blocked with 5\% skimmed milk in Tris-buffered saline (TBS) buffer at room temperature for $1 \mathrm{~h}$ and then incubated with primary antibodies (HMGB 1 1:1000, RAGE 1:800, NF-kB p65 1:1000, GAPDH 1:1500, H3 1:1500) overnight at $4{ }^{\circ} \mathrm{C}$. HMGB 1, RAGE, and NF-kB p65 were purchased from Abcam (Cambridge, UK, Ab18256, Ab3611, Ab16502). GAPDH, and H3 were purchased from Cell Signaling Technology (MA, USA, \#5471, \# 8173). After rinsing with TBS-T (10 $\min \times 3$ ), the membranes were incubated with an HRP-conjugated anti-rabbit antibody (1:1000, Beyotime, Shanghai, China, Ab18256) at room temperature for $1 \mathrm{~h}$. Finally, the membranes were washed three times, and the immunoreactive bands were visualized using ECL Plus (Life Technology Corporation, Gaithersburg, MD, USA) with a ChemiDoc XRS bioimaging system (Bio-Rad Laboratories, Inc., Hercules, CA, USA). 


\section{Statistical Analysis}

All values were expressed as the mean \pm SEM. The statistical analysis was performed using a one-way analysis of variance (ANOVA) by GraphPad Prism 7.0 software. A value of $p<0.05$ was considered statistically significant.

\section{Results}

\section{Impacts of MLDL on STC-Induced Pancreatic Injury}

Representative histopathological changes of pancreatic tissues were obtained from rats of different groups. Figure 1a shows that in the SO group, the histological examination revealed a normal architecture without obvious alteration in tissue morphology. However, the pancreas of SAP rats exhibited characteristic pathological alterations, such as inflammatory cell infiltration, focal necrosis, interstitial edema, and hemorrhage. The MLDL group exhibited relatively mild pathological injury, which manifested as a more integrated lobule structure and mild edema with infiltration of fewer inflammatory cells. The pancreatic pathological score of the SAP group was significantly higher compared with the SO group $(p<0.001)$. Figure $1 \mathrm{~b}$ shows that there was a significant reduction in the pancreatic pathological score in rats pretreated with MLDL compared with SAP rats $(p<0.05)$.

\section{Impacts of MLDL on SAP-Associated Lung Injury}

We also evaluated the severity of lung injury associated with SAP. Lung tissues from the SO group exhibited normal structures without histological modifications under a light microscope (Fig. 2a). However, lung tissues from the SAP group showed severe histologic lesions, including a widespread increase in alveolar wall thickness caused by edema, pulmonary interstitial hyperemia, hemorrhage, and apparent infiltration of inflammatory cells, indicating that the ALI model was successfully induced by SAP. However, the lung tissues of MLDL rats showed reduced interstitial edema and decreased infiltration of inflammatory cells compared with $\mathrm{SAP}$ rats. Besides, there were significant differences in the pathological score between the SAP group and the SO group $(p<0.001)$, as well as between the MLDL group and the SAP group ( $p<0.05$; Fig. 2b).

Next, we assessed the severity of inflammation by measuring the lung MPO activity, which was detected as an indicator of neutrophil sequestration into the tissues. In the present study, the lung MPO activity was low in the SO group, and it was significantly increased at $24 \mathrm{~h}$ after the induction of SAP $(p<0.001)$. Rats receiving MLDL demonstrated a significant reduction in MPO activity $(p<0.05$; Fig. $2 \mathrm{c})$.
A

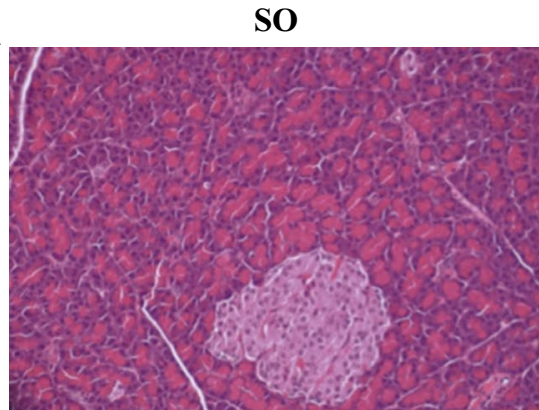

SAP

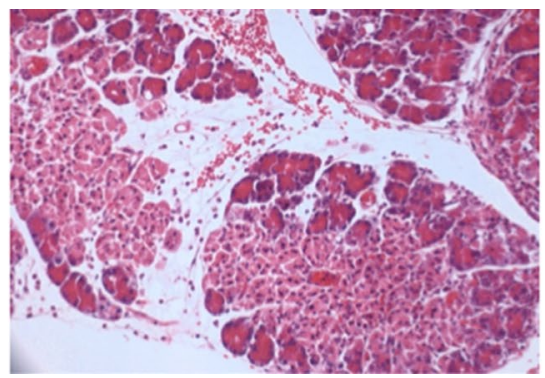

B

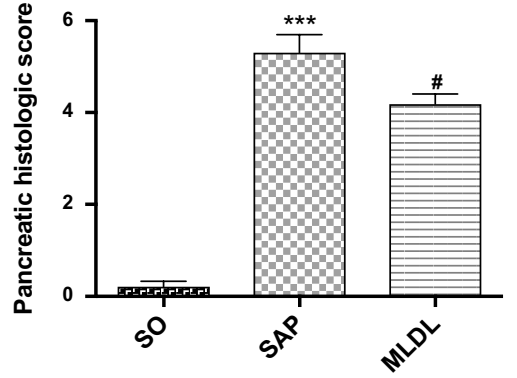

MLDL

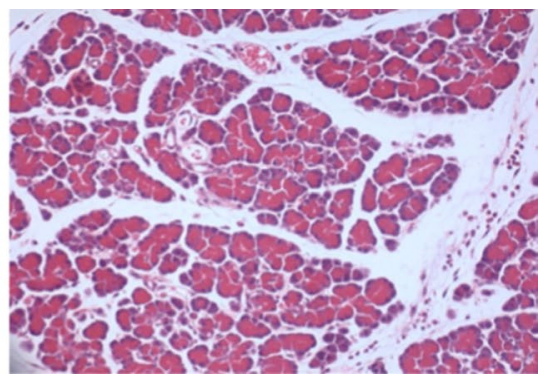

experimental group. $* * * p<0.001$, compared with the SO group; $\# p<0.05$, compared with the SAP group. $n=10-14$

Fig. 1 Impacts of MLDL on STC-induced pancreatic injury. a Images of H\&E staining, $200 \times$ magnifications of pancreas tissues of each experimental group. b Pathological score of pancreas tissues of each 

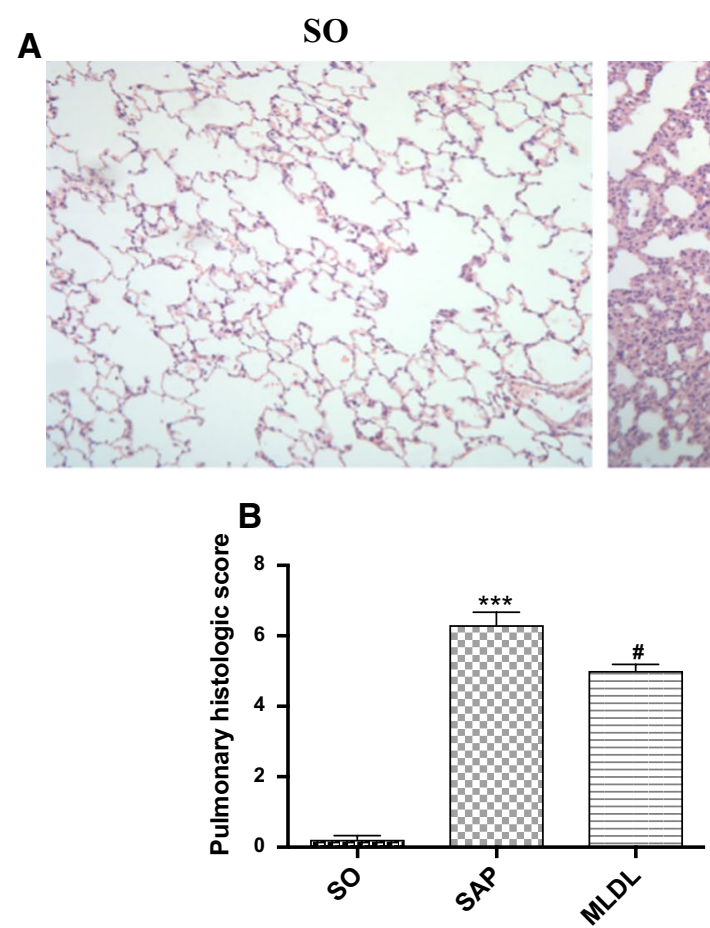

Fig. 2 Impacts of MLDL on SAP-associated lung injury. a Images of H\&E staining, $200 \times$ magnifications of lung tissues of each experimental group. b Pathological score of lung tissues of each experi-

\section{Impacts of MLDL on SAP-Associated Intestinal Injury}

The intestinal mucosa of SO rats showed good morphology (Fig. 3a). There were serious injuries in the intestinal mucosa of SAP rats. For example, the intestinal mucosa showed congestion and edema, the villi became short, shed and defected, accompanied by inflammatory cell infiltration. However, these histological damages were not significantly improved in MLDL rats compared with SAP rats. Consistent with the morphological changes of the mucosa, the histological scores of the SAP group were significantly higher compared with the SO group $(p<0.001)$, while there was no significant difference between the SAP and the MLDL groups ( $p>0.05)$ (Fig. 3b).

The D-LA and DAO levels were detected to judge the degree of the intestinal barrier injury. Figure $3 c$, d reveals that rats in the SO group had the lowest serum D-LA and DAO levels. In contrast, the levels of D-LA and DAO in the SAP group were markedly higher compared with the SO group. Additionally, MLDL intervention could significantly reduce the serum D-LA and DAO levels compared with the SAP group $(p<0.05)$.
SAP

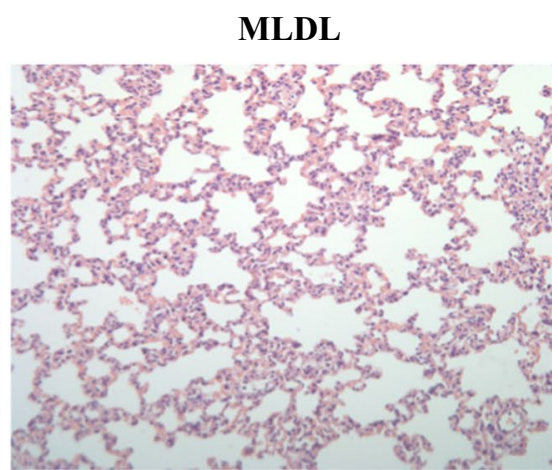

C

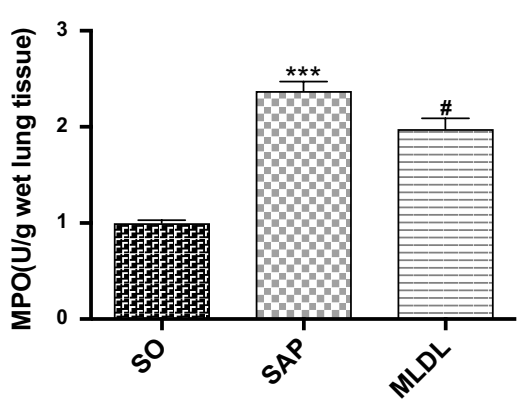

mental group. c MPO activity in lung tissues of each experimental group. $* * * p<0.001$, compared with the SO group; $\# p<0.05$, compared with the SAP group. $n=10-14$

\section{Impacts of MLDL on the Expressions of HMGB1, RAGE, and NF-KB p65 at the mRNA and Protein Levels in Lung Tissues}

RT-PCR and Western blotting analysis were performed to investigate whether MLDL had any effect on the expressions of HMGB1 and its downstream signaling in the lung (Fig. 4a). The results demonstrated that the HMGB 1 activity was increased in the lung of SAP rats, which could be counteracted by MLDL. Meanwhile, the expression of RAGE at the mRNA level in the lung of SAP rats was significantly increased, and such an increase could be effectively inhibited by MLDL. Besides, SAP rats also showed an increased expression of NF- $\mathrm{kB}$ p65 at the mRNA level, and MLDL could also inhibit such up-regulation. Moreover, Western blotting analysis indicated that the expressions of HMGB1, RAGE, and NF- $\mathrm{KB}$ p65 at the protein levels in the lung tissue were significantly elevated in the SAP group compared with the SO group $(p<0.001)$. However, Fig. $4 c$ shows that the expressions of HMGB1, RAGE, and NF- $\mathrm{kB}$ p65 at the protein level in the MLDL group were significantly decreased compared with the SAP group $(p<0.05)$. 

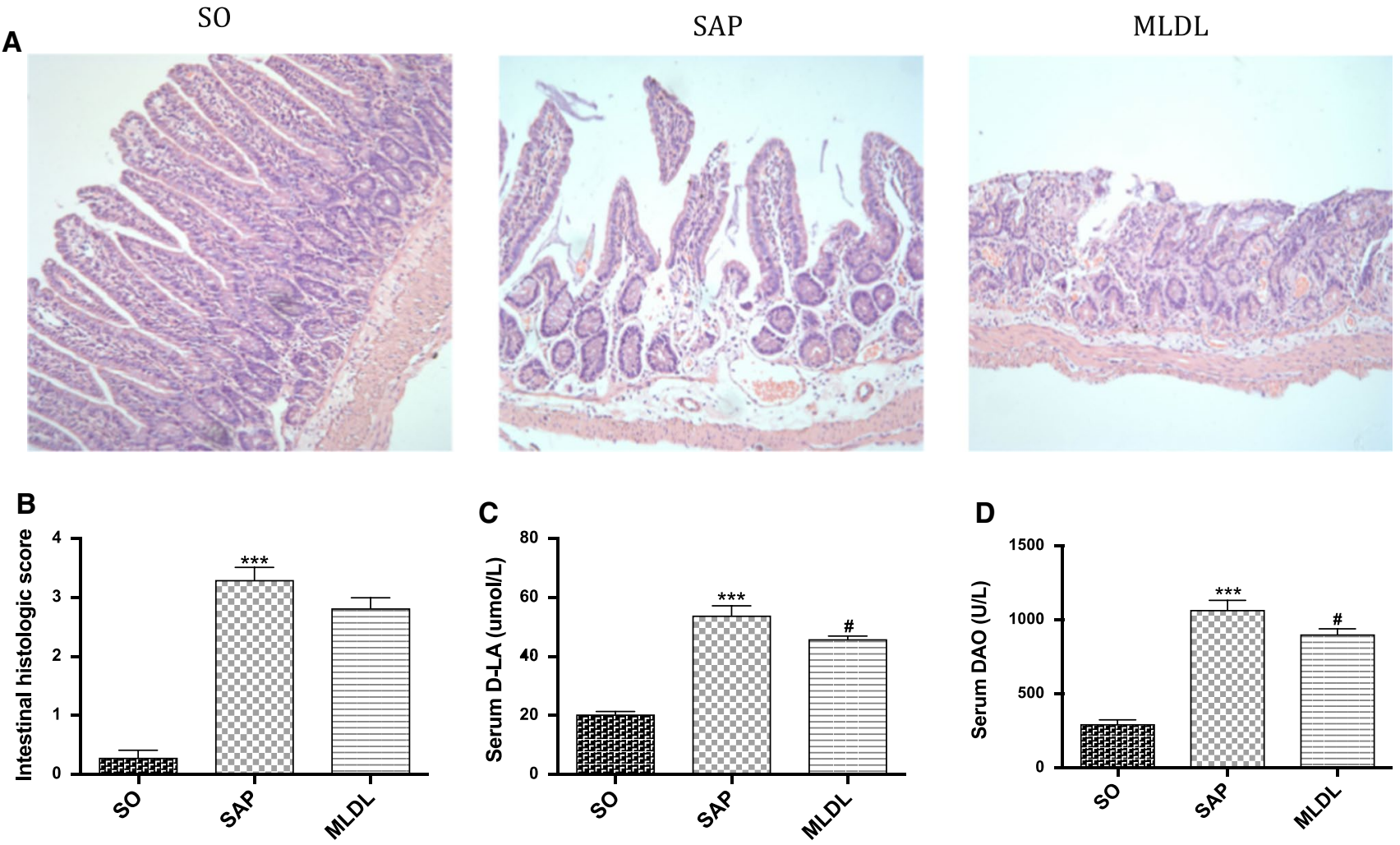

Fig. 3 Impacts of MLDL on SAP-associated intestinal injury. a Images of intestinal tissues $\mathrm{H} \& \mathrm{E}$ staining of each experimental group $(200 \times$ magnifications). b Pathological score of intestinal tissues of each experimental group. c Serum D-LA levels of each experi- mental group. d Serum DAO levels of each experimental group. $* * * p<0.001$, compared with the SO group; $\# p<0.05$, compared with the SAP group. $n=10-14$

\section{Impacts of MLDL on the Levels of Inflammatory Factors in Serum and Intestinal Lymphoid Tissues}

The concentrations of inflammatory factors in serum, including TNF- $\alpha$, IL-6, and ICAM1, were determined by ELISA. The serum levels of TNF- $\alpha$, IL-6, and ICAM1 in the SO group were the lowest, while these indices were significantly increased in the SAP group $(p<0.001)$. Compared with the SAP group, the levels of the above-mentioned three cytokines in the MLDL group were significantly reduced $(p<0.05)$ (Fig. 5a).

Next, we explored whether MLDL affected the expressions of HMGB1, RAGE, and TNF- $\alpha$ at the mRNA level in intestinal lymphoid tissues. Figure $5 b$ shows that the expressions of HMGB1, RAGE, and TNF- $\alpha$ in the SAP group were all significantly increased $(p<0.001)$ compared with the SO group. However, they all distinctly decreased in the MLDL group $(p<0.05)$ compared with the SAP group.

\section{Discussion}

ALI is the most common type of organ failure in SAP, and it is associated with high mortality. The release of gutderived injurious factors into the mesenteric lymph has been implicated in the development of ALI [28]. Therefore, we preliminarily aimed to explore the effect of blockade of mesenteric lymph flow by MLDL on SAP-related ALI. Our findings mainly included: (1) during SAP, MLDL could alleviate pancreatic and lung injury, but could not improve the pathological damage of the intestine; (2) MLDL could inhibit the production of inflammatory cytokines, such as TNF- $\alpha$, IL-6, and ICAM1; and (3) the mechanism of MLDL underlying the down-regulation of cytokine production and against SAP-ALI involved inhibition of HMGB1/RAGE/ $\mathrm{NF}-\kappa \mathrm{B}$ signaling. Collectively, these findings provided novel insights into the roles and mechanisms of mesenteric lymph in SAP-ALI. 

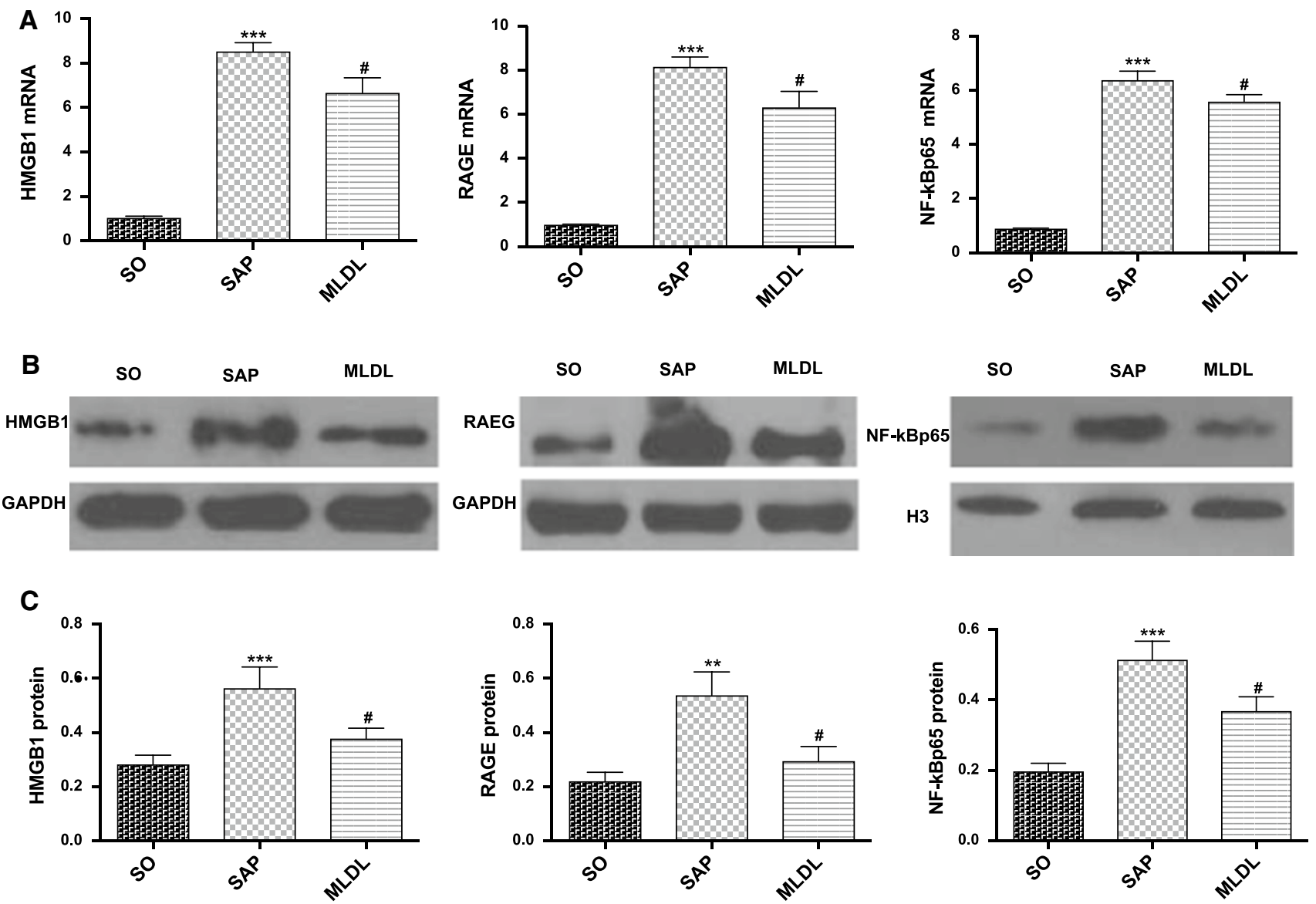

Fig. 4 Impacts of MLDL on the expressions of HMGB1, RAGE, and NF- $\kappa B$ p65 at the mRNA and protein levels in lung tissues. a RTPCR analysis of HMGB1, RAGE, and NF- $\mathrm{kB}$ p65 mRNA expression in lung tissues. b Western blot results of HMGB1, RAGE, and

Studies on both animal models and human beings have documented that gut permeability is increased shortly after the onset of pancreatitis [29-32]. Gut barrier dysfunction has been detected in $60 \%$ of AP patients [33]. The recession of intestinal barrier is associated with the development of SIRS and MODS, which can be attributed to the translocation of bacteria, toxins, and pro-inflammatory factors [30]. Therefore, it may be an effective therapeutic regime for SAP-ALI, by repairing intestinal barrier and/or reducing the spread of injurious factors released from impaired intestine.

It has been well documented that there are two draining systems in the gut, including portal vein and mesenteric lymph ducts $[34,35]$. The portal vein had previously regarded as the major conduit, while a great deal of evidence indicates that the mesenteric lymph ducts function as an equally critical pathway in the delivery of detrimental mediators into the systemic circulation, by which the intestine affects distant organs, including the lung $[36,37]$. Some animal studies have demonstrated that the interrupted flow of mesenteric lymph can ameliorate
NF- $\kappa$ B p65 protein expression in lung tissues. c Statistical results of B scanning densitometry. $* * * p<0.001$, compared with the SO group; $\# p<0.05$, compared with the SAP group. $n=10-14$

the distant organ failure $[12,38]$. Experimental studies on shock, trauma, burn, as well as ischemia/reperfusion (I/R) models all support the concept that lung injury can be prevented by ligation of the mesenteric lymph ducts [36, 39, 40]. However, its role in SAP-ALI remains largely unexplored. Thus, we aimed to assess the impacts and potential mechanism of MLDL on experimental SAP-ALI.

First, a preliminary experiment was carried out to ensure whether the intestinal mucosal injury could be caused by MLDL. We ligated the mesenteric lymph ducts of normal rats and compared them with non-ligated rats. No obvious morphological changes and inflammatory cell infiltration of the intestinal mucosa were observed by H\&E staining at $24 \mathrm{~h}$ after the operation (Fig. S1). This finding was consistent with the latest study that blockade of mesenteric lymph flow during intestinal I/R does not change the neutrophil infiltration in local gut tissues [41]. Nevertheless, it is unclear whether long-term MLDL will cause intestinal mucosal damage. 

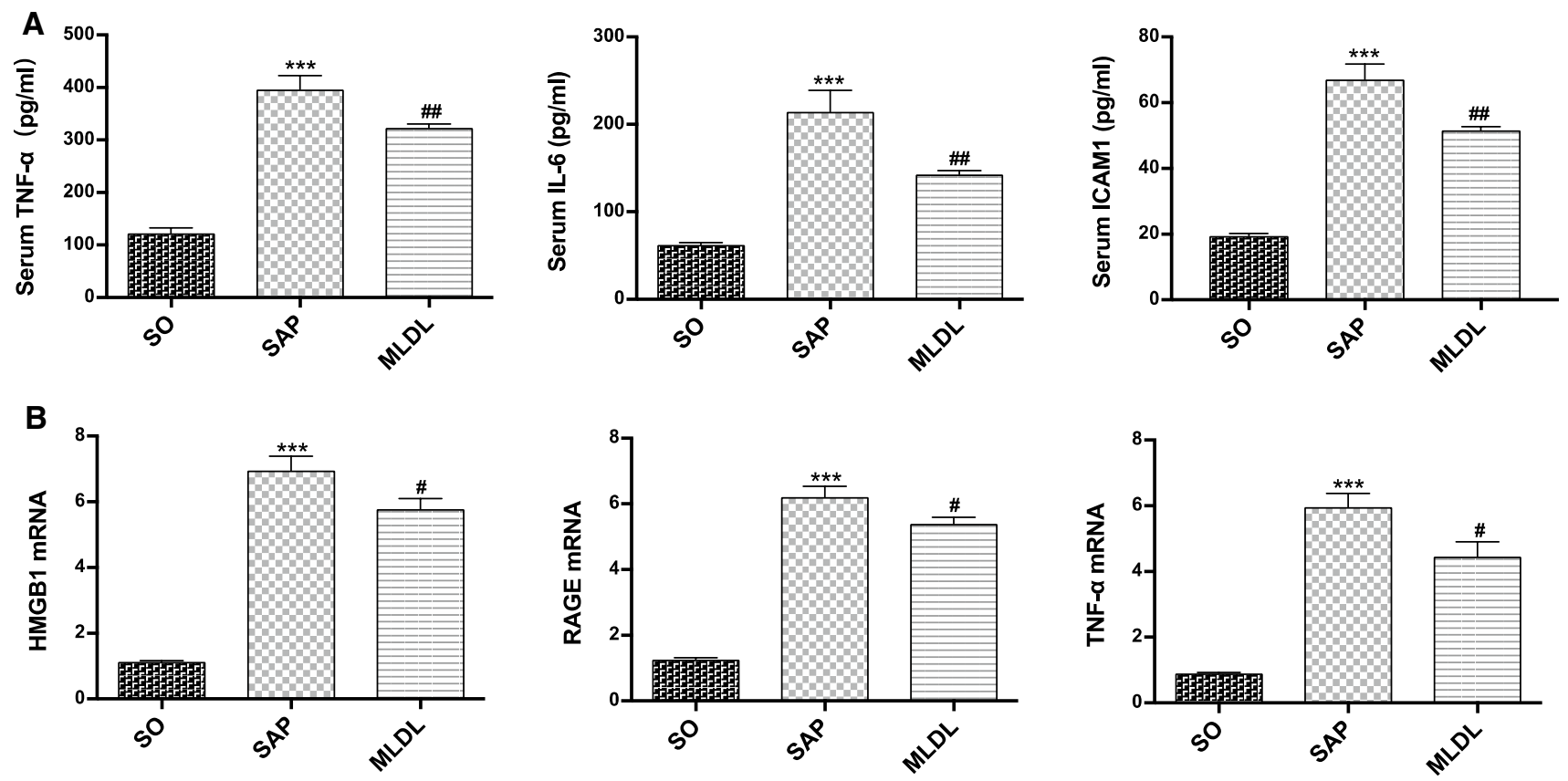

Fig. 5 Impacts of MLDL on the levels of inflammatory factors in serum and intestinal lymphoid tissues. a ELISA data of TNF- $\alpha$, IL-6 and ICAM1 in serum; b RT-PCR analysis of HMGB1, RAGE and

In the present study, the SAP-ALI model was successfully established by retrograde injection of 5\% STC into the biliopancreatic duct and verified by H\&E staining. In SAP rats, profound pancreatic injury accompanied by severe pulmonary injury was demonstrated at $24 \mathrm{~h}$ after modeling. Interestingly, the pathologic damages were alleviated by MLDL both in the pancreas and lung, suggesting the protective effect of MLDL against SAP-ALI.

The histologic changes of the intestine were assessed by H\&E staining. We found that intestines from the SAP rats were severely damaged. However, we failed to identify significant improvements in the pathological changes in the MLDL group. In Anthony's study, the intestinal injury caused by intraperitoneal injection of LPS is also not abrogated by MLDL, while the lung injury and neutrophil activation are reduced [42]. Similar results have been observed in He's study, suggesting that its thoracic duct drainage rather than ligation attenuates the intestinal injury, accompanied by lung and pancreatic renovation in rats with acute hemorrhagic necrotizing pancreatitis (AHNP) [43]. Both our and their findings showed that MLDL before SAP modeling might have no protective impacts on the gut morphology. The lung injury in MLDL + SAP rats might not be attenuated through improving gut injury.

DAO and D-LA are two sensitive indicators reflecting intestinal mucosal integrity and permeability. Consistent with previous results, we also found that the elevated serum DAO and D-LA levels were decreased by MLDL [43].
TNF- $\alpha$ mRNA in intestinal lymphoid tissues. $* * * p<0.001$, compared with the SO group; $\# p<0.05$, \#\#p $<0.01$, compared with the SAP group. $n=10-14$

Nevertheless, there seemed to be a contradiction that the intestinal histopathological damage was not improved, while the index reflecting the intestine damage in the blood was ameliorated. Since the mesenteric lymph can transport gutderived factors into the blood circulation, a reasonable interpretation for such discrepancy might be attributed to MLDL partly blocked the access of DAO and D-LA to the blood.

It has also been suggested that ALI commonly involves the excessive generation and release of multiple inflammatory cytokines [44]. Therefore, elucidation of the underlying molecular mechanisms associated with inflammation and methods featuring anti-inflammatory activity may be beneficial for ALI. HMGB1 binding to RAGE has been shown to augment inflammatory responses by activating NF- $\kappa B$, playing a crucial role in AP [45, 46]. Inhibition of HMGB1 and its downstream signaling has beneficial effects on SAP development in rats [47]. Studies have also demonstrated that HMGB1-dependent activation of NF- $\mathrm{\kappa B}$ is implicated in the development of ALI [47]. However, it remains largely unexplored whether the HMGB1/RAGE/NF- $\kappa$ B signaling plays a significant role in the pathogenesis of SAP-induced ALI. Using the well-established SAP-ALI model, we demonstrated that the expressions of HMGB1, RAGE, and $\mathrm{NF}-\kappa \mathrm{B}$ p56 at the mRNA and protein levels in lung and intestinal lymphoid tissues were significantly higher in SAP rats compared with the SO rats, and such up-regulation could be reversed by MLDL. Additionally, the expressions of inflammatory cytokines, including TNF- $\alpha$, IL- 6 , and 
ICAM1, were significantly elevated in the serum of SAP rats. Pretreatment with MLDL reduced such up-regulation and amended the histopathological injury of lung. Thence, MLDL could decrease the release of pro-inflammatory cytokines by blocking the HMGB1/RAGE/NF- $\mathrm{\kappa B}$ signaling.

Mesenteric lymph draining from gut contains several biologically active agents, including electrolytes, lipids, proteins, and immune cells [48]. During acute and critical illness, the composition of mesenteric lymph is significantly changed [37]. A previous investigation has shown that the lymph concentrations of TNF- $\alpha$, IL- 6 , and ICAM-1 are increased [49]. It is well known that HMGB1 is secreted by necrotic cells or activated monocytes and macrophages following stimulation with LPS, or inflammatory factors, such as TNF- $\alpha$, or IL- $1 \beta$ [50]. Extracellular HMGB1 plays a pro-inflammatory role, and it perpetuates inflammatory reactions in a wide variety of pathologies. Therefore, we hypothesized that local gut tissues injury caused the release of bioactive agents into the mesenteric lymph during SAP, followed by the distribution in the systemic and pulmonary blood circulation, where mesenteric lymph-derived factors stimulate HMGB1 secretion and induced lung damage via NF- $\kappa B$ signaling. MLDL blocked the major pathway of gut-derived lymphotoxic substances to the lung, leading to reduced HMGB 1 release and alleviated pulmonary inflammation.

In conclusion, our findings confirmed that mesenteric lymph was involved in the pathogenesis of SAP-ALI. MLDL could alleviate SAP-ALI probably by inhibiting HMGB1induced inflammation, which originated from the injured intestine in rats. These findings further elucidated the mechanism underlying SAP-ALI and further highlighted the importance of protecting intestinal barrier. Collectively, the modulation of mesenteric lymph flow might provide opportunities to develop new strategies to counteract the development of SAP-ALI.

Acknowledgments We thank the National Natural Science Foundation of China (Nos.81302929, 81973809), National Traditional Chinese Medicine Innovation Backbone Talents Training Project, and Science and Technology Innovation Action Plan of Science and Technology Commission of Shanghai Municipality (No. 19401971800) for funding this project.

\section{Compliance with Ethical Standards}

Conflict of interest The authors declare no conflicts of interest.

Open Access This article is licensed under a Creative Commons Attribution-NonCommercial 4.0 International License, which permits any non-commercial use, sharing, adaptation, distribution and reproduction in any medium or format, as long as you give appropriate credit to the original author(s) and the source, provide a link to the Creative Commons licence, and indicate if changes were made. The images or other third party material in this article are included in the article's Creative Commons licence, unless indicated otherwise in a credit line to the material. If material is not included in the article's Creative Commons licence and your intended use is not permitted by statutory regulation or exceeds the permitted use, you will need to obtain permission directly from the copyright holder. To view a copy of this licence, visit http://creativecommons.org/licenses/by-nc/4.0/.

\section{References}

1. Sternby H, Bolado F, Canaval-Zuleta HJ et al. Determinants of severity in acute pancreatitis: a nation-wide multicenter prospective cohort study. Ann Surg. 2019;270:348-355

2. Van Dijk SM, Hallensleben NDL, van Santvoort HC et al. Acute pancreatitis: recent advances through randomised trials. Gut. 2017;66:2024-2032

3. Johnson CD, Abu-Hilal M. Persistent organ failure during the first week as a marker of fatal outcome in acute pancreatitis. Gut. 2004;53:1340-1344

4. Huang L, Wang $\mathrm{MH}$, Yang $\mathrm{XN}$ et al. Acute lung injury in patients with severe acute pancreatitis. Turk J Gastroenterol. 2013;24:502-507

5. Zhou MT, Chen CS, Chen BC et al. Acute lung injury and ARDS in acute pancreatitis: mechanisms and potential intervention. World J Gastroenterol. 2010;16:2094-2099

6. Akbarshahi H, Rosendahl AH, Westergren-Thorsson $\mathrm{G}$ et al. Acute lung injury in acute pancreatitis-awaiting the big leap. Respir Med. 2012;106:1199-1210

7. Shields CJ, Winter DC, Redmond HP. Lung injury in acute pancreatitis: mechanisms, prevention, and therapy. Curr Opin Crit Care. 2002;8:158-163

8. Liu J, Huang L, Luo M et al. Bacterial translocation in acute pancreatitis. Crit Rev Microbiol. 2019;45:539-547

9. Mole DJ, Olabi B, Robinson V et al. Incidence of individual organ dysfunction in fatal acute pancreatitis: analysis of 1024 death records. $H P B($ Oxford $)$. 2009;11:166-170

10. Garg PK, Singh VP. Organ failure due to systemic injury in acute pancreatitis. Gastroenterology. 2019;156:2008-2023

11. Deitch EA, Xu D, Kaise VL. Role of the gut in the development of injury- and shock induced SIRS and MODS: the gut-lymph hypothesis, a review. Front Biol. 2006;11:520-528

12. Adams CA, Hauser CJ, Adams JM et al. Trauma-hemorrhageinduced neutrophil priming is prevented by mesenteric lymph duct ligation. Shock. 2002;18:513-517

13. Deitch EA, Adams C, Lu Q et al. A time course study of the protective effect of mesenteric lymph duct ligation on hemorrhagic shock-induced pulmonary injury and the toxic effects of lymph from shocked rats on endothelial cell monolayer permeability. Surgery. 2001;129:39-47

14. Zhang H, Zhai JY, Du HB et al. Mesenteric lymph drainage alleviates hemorrhagic shock-induced spleen injury and inflammation. Acta Cir Bras. 2019;34:e201900903

15. Sambol J, Deitch EA, Takimoto K et al. Cellular basis of burninduced cardiac dysfunction and prevention by mesenteric lymph duct ligation. J Surg Res. 2013;183:678-685

16. Sambol JT, Lee MA, Jiang M et al. Mesenteric lymph from rats with trauma-hemorrhagic shock causes abnormal cardiac myocyte function and induces myocardial contractile dysfunction. $J$ Appl Physiol. 2011;111:799-807

17. Yang H, Wang H, Andersson U. Targeting inflammation driven by HMGB1. Front Immunol. 2020;11:484

18. Jakkampudi A, Jangala R, Reddy BR et al. NF- $\kappa B$ in acute pancreatitis: Mechanisms and therapeutic potential. Pancreatology. 2016; $16: 477-488$ 
19. Malarkey CS, Churchill ME. The high mobility group box: the ultimate utility player of a cell. Trends Biochem Sci. 2012;37:553-562

20. Yu Y, Tang D, Kang R. Oxidative stress-mediated HMGB1 biology. Front Physiol. 2015;6:93

21. Shen $X$, Li WQ. High-mobility group box 1 protein and its role in severe acute pancreatitis. World J Gastroenterol. 2015;21:1424-1435

22. Arriaga-Pizano L, Bosco-Garate I, Martinez-Ordaz JL et al. High serum levels of high-mobility group box 1 (HMGB1) and low levels of heat shock protein 70 (Hsp70) are associated with poor prognosis in patients with acute pancreatitis. Archive Med Res. 2018;49:504-511

23. Choi SB, Bae GS, Park KC et al. Opuntia humifusa ameliorated cerulein-induced acute pancreatitis. Pancreas. 2014;43:118-127

24. Hagiwara S, Iwasaka H, Uchida T et al. Danaparoid sodium prevents cerulein-induced acute pancreatitis in rats. Shock. 2009;32:94-99

25. Kubisch C, Dimagno MJ, Tietz AB et al. Overexpression of heat shock protein Hsp27 protects against cerulein-induced pancreatitis. Gastroenterology. 2004;127:275-286

26. Imanaka $\mathrm{H}$, Shimaoka $\mathrm{M}$, Matsuura $\mathrm{N}$ et al. Ventilator-Induced lung injury is associated with neutrophil infiltration, macrophage activation, and TGF- $\beta 1$ mRNA upregulation in rat lungs. Anesth Analg. 2001;92:428-436

27. Crissinger K. Animal models of necrotizing enterocolitis. J Pediatr Gastroenterol Nutr. 1995;20:17-22

28. Ma Y, Yang X, Chatterjee V, et al. The gut-lung axis in systemic inflammation: role of mesenteric lymph as conduit. Am J Respir Cell Mol Biol. 2020. https://doi.org/10.1165/rcmb.2020-0196TR

29. Fishman JE, Levy G, Alli V et al. The intestinal mucus layer is a critical component of the gut barrier that is damaged during acute pancreatitis. Shock. 2014;42:264-270

30. Schietroma M, Pessia B, Carlei F et al. Intestinal permeability and systemic endotoxemia in patients with acute pancreatitis. Ann Ital Chir. 2016;87:138-144

31. Liang HY, Chen T, Wang T et al. Time course of intestinal barrier function injury in a sodium taurocholate-induced severe acute pancreatitis in rat model. J Dig Dis. 2014;15:386-393

32. Koh YY, Yong KC, Hong JK et al. The effect of intestinal permeability and endotoxemia on the prognosis of acute pancreatitis. Gut Liver. 2012;6:505-511

33. Wu LM, Sankaran SJ, Plank LD et al. Meta-analysis of gut barrier dysfunction in patients with acute pancreatitis. Br J Surg. 2014;101:1644-1656

34. Breslin JW, Yang Y, Scallan JP et al. Lymphatic vessel network structure and physiology. Compr Physiol. 2018;9:207-299

35. Glaser T, Baiocchi L, Zhou T et al. Pro-inflammatory signalling and gut-liver axis in nonalcoholic and alcoholic steatohepatitis: differences and similarities along the path. J Cell Mol Med. 2020;24:5955-5965

36. Adams CJ, Sambol JT, Xu DZ et al. Hemorrhagic shock induced up-regulation of P-selectin expression is mediated by factors in mesenteric lymph and blunted by mesenteric lymph duct interruption. J Trauma. 2001;51:625-631

37. Deitch EA. Gut lymph and lymphatics: a source of factors leading to organ injury and dysfunction. Ann NY Acad Sci. 2010;1207:E103-E111. https://doi.org/10.111 1/j.1749-6632.2010.05713.x

38. Cavriani G, Domingos HV, Soares AL et al. Lymphatic system as a path underlying the spread of lung and gut injury after intestinal ischemia/reperfusion in rats. Shock. 2005;23:330-336

39. Tong $\mathrm{H}$, Chen $\mathrm{R}$, Yin $\mathrm{H}$ et al. Mesenteric lymph duct ligation alleviating lung injury in heatstroke. Shock. 2016;46:696-703

40. He GZ, Dong LG, Chen XF et al. Lymph duct ligation during ischemia/reperfusion prevents pulmonary dysfunction in a rat model with omega-3 polyunsaturated fatty acid and glutamine. Nutrition. 2011;27:604-614

41. Ma Y, Zabell T, Creasy A et al. Gut ischemia reperfusion injury induces lung inflammation via mesenteric lymph-mediated neutrophil activation. Front Immunol. 2020;11:586685

42. Watkins AC, Caputo FJ, Badami C et al. Mesenteric lymph duct ligation attenuates lung injury and neutrophil activation after intraperitoneal injection of endotoxin in rats. $J$ Trauma. 2008;64:126-130

43. Peng H, Zhi FW, Su MJ et al. Blocking abdominal lymphatic flow attenuates acute hemorrhagic necrotizing pancreatitis-associated lung injury in rats. J Inflamm (Lond). 2013;10:9

44. Zhang H, Neuhöfer P, Song L et al. IL-6 trans-signaling promotes pancreatitis-associated lung injury and lethality. J Clin Invest. 2013;123:1019-1031

45. Li G, Wu X, Yang L et al. TLR4-mediated NF- $\kappa$ B signaling pathway mediates HMGB1-induced pancreatic injury in mice with severe acute pancreatitis. Int J Mol Med. 2016;37:99-107

46. Yang R, Tenhunen J, Tonnessen TI. HMGB1 and histones play a significant role in inducing systemic inflammation and multiple organ dysfunctions in severe acute pancreatitis. Int $J$ Inflam. 2017;2017:1817564

47. Zhao S, Yang J, Liu T et al. Dexamethasone inhibits NFsmall ka, CyrillicBp65 and HMGB1 expression in the pancreas of rats with severe acute pancreatitis. Mol Med Rep. 2018;18:5345-5352

48. Fanous MY, Phillips AJ, Windsor JA. Mesenteric lymph: the bridge to future management of critical illness. Jop. 2007:8:374-399

49. Zhang Y, Zhang S, Tsui N. Mesenteric lymph duct drainage attenuates acute lung injury in rats with severe intraperitoneal infection. Inflammation. 2015;38:1239-1249

50. Van Gorp H, Van Opdenbosch N, Lamkanfi M. Inflammasomedependent cytokines at the crossroads of health and autoinflammatory disease. Cold Spring Harb Perspect Biol. 2019;11:a028563

Publisher's Note Springer Nature remains neutral with regard to jurisdictional claims in published maps and institutional affiliations. 